\title{
Saber y totalidad en Levinas y Foucault
}

\section{Gómez Florencia Andrea}

Universidad Nacional del Nordeste

\section{Resumen}

La cuestión del nexo saber-totalidad corresponde a una temática que se puede entrever en el pensamiento de Emmanuel Levinas y Michel Foucault. Si bien representan posturas diferentes con respecto a los modos de relación intersubjetivos, ambos se sumergen en la comprensión y cuestionamiento de la formación de un saber totalizante. Sus nociones rompen con la razón instrumental del mundo occidental. En relación a ello, Levinas anuncia el distanciamiento con respecto a ese pensar, que comprende las relaciones humanas meramente como relaciones de interés, proclamando en consecuencia otra forma de comprender al ser humano. Rompiendo con un paradigma esencialista, critica a éste como un modo que crea categorías universales cuasi axiomáticas. El saber que se encuentra atravesado por esa consideración de la objetividad científica, presenta al otro como un estante, como un ser a la mano, tal como diría Heidegger, asimilable y reductible a categorías del pensamiento.

Foucault analiza la anterior cuestión, a partir de la relación saber-poder. Uno de los mecanismos que ramifica su incidencia en todas las relaciones que se manifiestan en la sociedad, es el saber. La acción de este saber sujetante, mediante la utilización del lenguaje, a través de una serie de modificaciones epistemológicas establece un binarismo, ese binarismo se traduce en normal/anormal, bueno/malo, sano/enfermo. Es a través de esa dualidad que opera una tipificación, contraria a todo dinamismo de la vida, con el fin de nominar, de construir lo nombrado y trastocar a una singularidad en un ser sujetado. Esta relación de poder que se establece entre el diferente y saberes institucionalizados, siendo uno de ellos el saber psiquiátrico, se establece a 
partir de mecanismos de normatización y a través de las formas de sujeción que operan localmente.

Tomando como central la conformación de un saber-poder psiquiátrico, tal como lo presenta Foucault, periodo que va del siglo XVIII al siglo XIX, se analizará siguiendo asimismo la filosofía levinasiana, aquellos mecanismos y formas de acceso la alteridad, que conforman un saber totalizante que opera mediante una homogeneización impugnando toda heterogeneidad humana.

Palabras clave: Saber, totalidad, alteridad, poder, diferencia.

\section{Abstract}

The question of the nexus of knowledge-totality corresponds to a theme that can be glimpsed in the thought of Emmanuel Levinas and Michel Foucault. Although they represent different positions regarding intersubjective modes of relation, both immerse themselves in the comprehension and questioning of the formation of totalizing knowledge. His notions break with the instrumental reason of the Western world. In relation to this, Levinas announces the distance with respect to that thinking, which comprises human relations merely as relations of interest, proclaiming consequently another way of understanding the human being. Breaking with an essentialist paradigm, he criticizes it as a mode that creates quasi-axiomatic universal categories. The knowledge that is crossed by this consideration of scientific objectivity presents the other as a shelf, as a being at hand, as Heidegger would say, assimilable and reducible to categories of thought.

Foucault analyzes the previous question, based on the knowledge-power relationship. One of the mechanisms that ramifies its incidence in all the relations that are manifested in the society, is the knowledge. The action of this gripping knowledge, through the use of language, through a series of epistemological modifications establishes a binarism, that binarism is translated into normal / abnormal, good / bad, healthy / sick. It is through this duality that a typification operates, contrary to all dynamism of life, in order to nominate, to build the named and to overturn a 
singularity in a being held. This relationship of power that is established between the different and institutionalized knowledge, one of them being psychiatric knowledge, is established through mechanisms of regulation and through the forms of subjection that operate locally.

Taking as central the formation of a psychiatric knowledge-power, as presented by Foucault, a period that goes from the eighteenth to the nineteenth century, will be analyzed following the philosophy of the Levinas, those mechanisms and forms of access to otherness, which make up a totalizing knowledge which operates by homogenizing all human heterogeneity.

Key words: knowledge, totality, otherness, power, difference.

\section{Introducción}

En el presente artículo se abordará el nexo saber-totalidad y sus implicancias en la relación otro-Otro, desde las perspectivas de Emmanuel Levinas y Michel Foucault, aplicado específicamente a la institución psiquiátrica.

En la filosofía levinasiana, el concepto de otredad se puede ubicar como: otro en tanto próximo y Otro en tanto presencia cosificada. Respecto a esto, el filósofo acentúa en Totalidad e infinito que, el otro no es un simple dato que se aferra y queda estático e inestable, no es un objeto capturable de una verdad que se concibe como si fuera una simple adecuación. A este otro no lo determino a partir de ser ni a partir del conocimiento, sino que él permanece intacto en su alteridad, es absoluto. Así, la responsabilidad reclama la conversión del para-sí en para-otro, lo cual significa anteponer la sociabilidad como vulnerabilidad y no como preponderancia de las formas del poder censurante de la diferencia.

En Foucault, la relación Otro-otro está atravesada por una relación de poder y resistencia. La Otredad representa, en el exterior, significaciones religiosas, culturales e históricas que ejercen un poder sobre el otro. Para el mencionado autor, el otro frente a los embates del Otro inscribe el ejercicio de la libertad en la resistencia, 
transformándose en un acto singular del sujeto que resiste como respuesta al ejercicio del poder sobre su cuerpo, sus afectos, sus acciones y actos.

Las nociones anteriormente mencionadas ofrecen un modelo para comprender como se establece la unión saber-totalidad. Para dicho abordaje se va a recurrir específicamente a Michel Foucault en El poder psiquiátrico y a Emmanuel Levinas en Totalidad e infinito y en De otro modo que ser o más allá de la esencia.

\section{Genealogía del poder psiquiátrico en Foucault}

En El poder psiquiátrico Michel Foucault analiza las transformaciones que se fueron produciendo en las prácticas psiquiátricas y consecuentemente en las instituciones psiquiátricas, comenzando con un discurso de Pinel hasta llegar a Charcot en el siglo XIX. La relación de estas prácticas con los discursos en torno a la locura, encuentran gran mutación a partir de lo que el autor presenta como poder de soberanía a un poder disciplinario, conformado en el siglo XIX.

El poder de soberanía implica una relación asimétrica entre el soberano y el súbdito. El primero sustrae productos, fuerza de trabajo y servicios a costa del segundo, que a la larga no son devueltos. Sin embargo, a través de una operación de reciprocidad, el soberano efectúa un gasto, ya sea como servicio o gasto de sus riquezas. Debido a que este tipo de poder resulta frágil, necesita de una constante reactualización a través de complementos de violencia ${ }^{39}$, es decir, necesita de una serie de gestos como señales de respeto, insignias, blasones.

Así también, las relaciones no son isotópicas, esto quiere decir, que las relaciones de soberanía se entrelazan unas con otras imposibilitando la clasificación y con ello la instauración de un sistema en el cual la jerarquía sea exhaustiva y unitaria.

Asimismo, el poder soberano difiere cuantitativamente en el objeto de aplicación. Es así, como se aplica a multiplicidades: a familias, usuarios. Sin embargo la falta de individualidad no se encuentra en la cima, si no que se destaca la singularidad del cuerpo del rey. Allí se puede percibir la individualidad que no se encuentra abajo.

\footnotetext{
${ }^{39}$ Cfr., FOUCAULT, Michel. El poder psiquiátrico. Fondo de Cultura Económica, Bs. As, 2014. p. 63.
} 
El poder disciplinario, implica una inversión. Se destaca una individualización abajo y un poder múltiple en la cima. Su objeto consiste entonces en aplicarse a singularidades somáticas a partir de un poder anónimo, sin rostro y múltiple. Consecuentemente, su efecto no consiste en asegurar la centralización de poder mediante la visibilización de un individuo, sino que su fin central consiste en, la docilidad y sujeción de los cuerpos. Esa finalidad se percibe en tanto que, el poder disciplinario se caracteriza, no por la sustracción de productos, fuerza de trabajo y servicios, sino, "por la captura exhaustiva del cuerpo, los gestos, el tiempo, el comportamiento del individuo. Es una captura del cuerpo y no del producto; es una captura del tiempo en su totalidad y no del servicio" ${ }^{40}$. A partir de esa fuerte individualización en la base se convierte en productora de cuerpos sujetos.

Asimismo, se destaca por un principio de omnivisibilidad, es decir, su poder no es intermitente, sino que su aplicación es constante e ininterrumpida. Se basa en un control constante e ininterrumpido de los cuerpos y sus comportamientos. El sujeto que se halla bajo ese poder, se encuentra continuamente observado. Este control ininterrumpido, tiene como designio la generación de un hábito, mediante el cual logre la autorreproducción. Asimismo ese control se apoya en la escritura, a partir de la cual puede registrar toda la información captable de los individuos.

Por último, los dispositivos disciplinarios son isotópicos, así, a cada elemento le corresponde un lugar determinado, por lo tanto, es posible establecer un sistema clasificable. Sin embargo, lo isotópico implica algo inclasificable e inasimilable. Es así como, implica la aparición de un "residuo", que representa exactamente que se encuentra por fuera de ese sistema. Este concepto de "residuo" se analizará en páginas posteriores para determinar la relación otro-Otro dentro de este dispositivo.

\section{El poder psiquiátrico como poder disciplinario y sus transformaciones}

El saber- poder del cual se viene mencionando, ha sufrido modificaciones a lo largo de los siglos, así también ya conformado dentro de un sistema disciplinario, se

\footnotetext{
${ }^{40}$ lbíd., p. 66.
} 
pueden observar transformaciones en esos dispositivos de poder como productores de enunciados y de los juegos de verdad en torno a ellos.

El poder disciplinario presenta una noción de la corporalidad, como algo regulable en todas sus dimensiones, en sus comportamientos, gestos, actividades. Implica un orden, una disciplina y regularidad que logra penetrar en ellos, hasta lograr la parasitación de los cuerpos.

Desde Pinel, el saber médico se presenta como necesita para una observación exacta de un orden, una disciplina. Es así como el orden disciplinario representa una condición para ese tipo de observación. La distribución del tiempo, los cuerpos, los gestos, los comportamientos y los discursos le permite esa relación de objetividad propia de la mirada médica. A partir de esa distribución se da la condición de la curación. Contrario a la concepción del siglo XVIII, la operación terapéutica parte de una noción distinta de locura. El loco ya no es aquel que se engaña, sino que se lo caracteriza por "la insurrección de las fuerzas, se desencadena en él cierta fuerza indominable" ${ }^{41}$. Esto es lo que caracteriza, aunque de formas diferentes, al furioso, a la manía sin delirio, a la manía, a la melancolía o monomanía. Partiendo de aquella consideración, la táctica asilar va a basarse en la localización de esa explosión de la fuerza. Lo nuclear va a ser caracterizar el desencadenamiento de la detonación de las fuerzas. Y partir de ello, la curación, se basa en el sometimiento de las fuerzas. Tal como lo describe Pinel:

\footnotetext{
La terapeútica de la locura es "el arte de subyugar y domesticar, por así decirlo, al alienado, poniéndolo bajo la estricta dependencia de un hombre que, por sus cualidades físicas y morales, tenga la capacidad de ejercer sobre él un influjo irresistible y modificar el encadenamiento vicioso de sus ideas. ${ }^{42}$
}

Foucault destaca la práctica de Pinel atravesada por la violencia, la cual no remite, tal como critica Foucault a una fuerza irracional, desequilibrada. La violencia determinada aquí, es resultado de un juego calculado, racional. Éste se sirve de

\footnotetext{
${ }^{41}$ Ibíd., p. 23.

42 Ibíd., p. 24.
} 
determinados instrumentos, con el fin de quebrantar la fuerza explosiva del alienado. La curación no requiere por parte del médico de un discurso de verdad, es decir, no requiere de una labor de diagnóstico, en cambio, se trata de un enfrentamiento, un choque de voluntades, una relación de fuerza entre el enfermo y el médico.

Una escena interesante que sirve para dar cuenta de ese paso del poder soberano a un poder disciplinar, es la escena que presenta Pinel, de la liberación de los enfermos. Con ello genera un pasaje de la violencia salvaje de las cadenas a la obediencia, el loco debe someterse a la voluntad del médico. Se procede así, el paso de una forma de poder que corresponde a la vieja forma de poder de soberanía a una relación de sujeción que es una relación de disciplina.

Así también una de las tácticas propias dentro del siglo XVIII, es la estrategia utilizada por medio de la medicación, ésta actúa "como medicación en el delirio y como terapéutica del delirio" ${ }^{43}$. A modo de ejemplificación, se encuentra el caso de la observación de Mason $\mathrm{Cox}^{44}$. En dicho caso, el saber médico procede a otorgar realidad al delirio con el objeto de que el enfermo acepte la intervención médica a fin de curarse de aquello que su delirio le provocaba. $Y$ a partir de esa medicación en el delirio, se logra la curación del delirio. A principios del siglo XIX, ese juego de verdad generado a partir de la intensificación del delirio será abolido.

De lo que se trata según Foucault, no es centrar la mirada en la institución, sino focalizar las disposiciones estratégicas desencadenantes de determinadas relaciones de fuerza dentro de la institución. Centrarse en esto, significa señalar conjuntamente el dispositivo de poder y discursos de verdad.

Cabe señalar que en el siglo XIX opera también una reconstrucción del modelo familiar, que luego va a encontrarse en desuso. Así se introduce como centro el papel de la familia en el sistema disciplinario. En su recorrido histórico por las instituciones, Foucault encuentra que el papel de la familia es de suma importancia para la implementación. Conforme a esto, la familia representa la célula elemental del poder

\footnotetext{
${ }^{43}$ Ibíd., p. 55.

${ }^{44}$ Cfr., FOUCAULT, Michel. El poder psiquiátrico. p. 52.
} 
soberano. Es en la figura del padre en que se concentra el ejercicio del poder soberano, ya que éste es un poder corporizado y no es anónimo. La familia funciona como una instancia de coacción que va a fijar de una forma permanente a los individuos a los dispositivos disciplinarios.

Cuando se produce un desajuste en la institución familiar en el 1850 y 1860 , el sistema disciplinario funciona como suplente que, intenta por medio de las instituciones disciplinarias asemejar los vínculos familiares.

Sin embargo, en el siglo XIX, como es el caso de Fodére ${ }^{45}$, hasta el siglo $\mathrm{XX}$, opera un precepto, que se basa en la no aceptación del modelo familiar como terapéutica del alienado. Éste funciona como el núcleo detonante de la alineación. Es así como van actuar tres principios: el principio del aislamiento; el principio de distracción o de disociación; la tercera razón relacionada al principio de aislamiento, es lo que Esquirol presenta como sospecha sintomática, la cual hace referencia a que el loco, al no comprender lo que sucede en él, busca la causa en su entorno, en lo que le rodea y sigue así la creencia, de que su mismo núcleo familiar es el origen de sus males.

Esquirol, afirma conforme a aquello que ya no es la familia o el modelo familiar lo que va a asegurar la curación, sino por el contrario, es el hospital en semejanza al panóptico de Bentham lo que cura. Es así como, "la disposición arquitectónica, la organización del espacio, el modo de circulación por él, el modo de observar y ser observado, todo eso, tiene de por sí valor terapéutico”46. Esa máquina panóptica instituye a partir de su omnivisibilidad, como se mencionó anteriormente, a la construcción de un tipo de hábito, que lleva a la autorreproducción del principio de distracción.

En este punto, se ve el paso del estatus del loco, ya no es aquel que atenta contra los derechos y los privilegios de una familia, sino que es alguien que atenta contra la sociedad, resulta peligroso para ella. El loco no se encuentra referido

\footnotetext{
${ }^{45}$ Cfr., FOUCAULT, Michel. El poder psiquiátrico. p. 118.

${ }^{46}$ Ibíd., p. 124.
} 
meramente al campo familiar, sino a un campo médico estatal. Como se percibirá, la conjunción saber y poder psiquiátrico bajo diferentes formas, instalan un discurso de verdad que se presenta como un discurso legítimo.

Foucault destaca una gran diferencia entre los discursos de la época clásica y el discurso vigente en el siglo XIX. En la época clásica, como se mencionó anteriormente, el loco es aquel que se encuentra sumido en una falsa creencia, en una ilusión, y conforme a ello el psiquiatra se coloca del lado del delirio. De modo contrario, en el siglo XIX el psiquiatra, se posiciona del lado de la realidad. Conforme a esto, Foucault señala que en este siglo, "el psiquiatra es un factor de intensificación de lo real y el agente de un sobrepoder de lo real, mientras que en la época clásica era, el agente de poder de irrealización de la realidad” ${ }^{47}$. Es así como, el psiquiatra ya no va a mirar por el lado de la verdad y el error, e intenta transformar en verdad ese error. Resulta una cuestión que deja de tener lugar central dentro de la cura. Actúa de acuerdo a una verdad gozada a partir de su propio status de ciencia psiquiátrica. A partir de esto, la práctica psiquiátrica da lugar a dos tipos de discursos. Un discurso nosológico o de clasificación, el cual presenta la locura como una serie de enfermedades mentales y un discurso anatomopatológico, que es la manera de encontrar un correlato material a la locura.

Estos dos discursos otorgan a la psiquiatría su cientificidad, y con ello un nivel de estabilidad, tal que sitúan al psiquiatra como el que posee la verdad. No existe criterio externo de corrección del error, sino que la corrección resulta algo interno. Así menciona Foucault el discurso de la ciencia psiquiátrica con respecto a ello, "soy dueña de la verdad, si no de la verdad en su contenido, al menos de todos los criterios de verdad" $^{\prime 8}$. Sin embargo, frente a esa seguridad surge un problema, el de la simulación o histeria. La mentira de la simulación fue el acto de insurrección de los locos frente al poder psiquiátrico. A partir de este suceso se desemboca una crisis. La crisis se refiere a la desestabilización del psiquiatra como poseedor de la verdad, ya que los simuladores obligan a éste a sumirse en el juego de verdad y mentira que le proponen.

\footnotetext{
${ }^{47}$ Ibíd., p. 156.

${ }^{48}$ Ibíd., p. 159.
} 
Así fue el problema de la cuestión de la verdad tuvo que ser replanteada dentro de la psiquiatría.

En el siglo XIX partiendo del discurso nosológico y anatomopatológico, se estable un proceso de cura a partir de cuatro mecanismos. En primera instancia, se encuentra el desequilibrio de poder, se trata de transferir o dejar a un lado el poder, del lado del psiquiatra. Mediante ese desequilibrio presenta una situación disimétrica entre él y el paciente, al cual se lo puede volver dócil y consecuentemente destruir su omnipotencia. En segundo lugar está, la reutilización del lenguaje, por medio del ingreso de determinados mecanismos, como el de la orden y la obediencia. En tercera instancia, el ordenamiento u organización de las necesidades, que tiene como consecuencia la aparición de nuevas necesidades, como es la necesidad de la libertad. $Y$ en cuarto lugar, el enunciado de la verdad, el cual tiene como objetivo que el enfermo diga la verdad. "La operación de verdad se habrá cumplido en el momento en que el enfermo se haya reconocido en esa identidad"49 ${ }^{4}$ El loco se ha curado entonces cuando se ha reconocido en una realidad individual que, cabe decir, corresponde a una realidad médica impuesta por un poder asilar. En relación a la cura, se establecen tres técnicas: el interrogatorio, como ya se mencionó previamente, mediante el cual se fijaba una identidad al individuo; la droga, que brindaba al médico la posibilidad de una reproducción de la locura y con ello una oportunidad de dominio sobre ella; y la hipnosis, como los demás, también se caracteriza por la delegación del poder del alienado al médico y por otorgar una absoluta autoridad a éste, ya que al dormir al enfermo, se logra controlar su comportamiento.

En tanto que el loco queda fundado como objeto de saber, el asilo, en tanto que dispositivo disciplinario, es también el lugar de formación de un cierto tipo de verdad. La construcción de nosologías y clasificaciones psiquiátricas, la cuestión del diagnóstico, la prueba de realidad y sus formas, aparecen aquí como un producto directo del sistema disciplinario. La verdad se produciría, según Foucault, gracias a múltiples coacciones, siendo un producto de la aplicación racional de normas,

\footnotetext{
${ }^{49}$ Ibíd., p. 191.
} 
prescripciones, procedimientos, como las que se mencionaron anteriormente. El sujeto queda así, inmerso es esa estructura asilar, que lo abarca, lo clasifica y lo instala en una categoría que adquiere el denominador de "residuo", ese remanente de la sociedad.

\section{Levinas: El Otro y el Mismo}

El filósofo lituano, en Humanismo del otro hombre, advierte la crisis del humanismo. Esa crisis tiene su raíz en la ineficacia o en la inconsistencia humana, que basa su concepción del sujeto como ser racional, y a partir de ello, se encuentra la consideración como ser dominador, poderoso frente a todo. Ante el fracaso de tal proyecto, señala Levinas que, asistiríamos a la ruina del mito del hombre como fin en sí, al dejar aparecer un orden, ni humano ni inhumano, un orden formal y abstracto. ${ }^{50}$

Levinas, precisa con aquello, la necesidad de otra mirada del ser humano, una salida de esa crisis, cuya concepción del sujeto se centraba en su poder, esto es, en el carácter iniciador de la conciencia. Pero, existe algo anterior a toda libertad y voluntad, anterior a todo poder del sujeto, que es la pasividad originaria, radical. Esto es posible gracias al Otro, que hace inquietar al Mismo. Le imputa una responsabilidad, que no ha surgido de una decisión tomada por el yo, sino de un principio anárquico, que precede al ser, y que es una pasividad insuperable. Y ello, debido a que, la responsabilidad antecede a toda intencionalidad.

Como se podrá percibir, uno de los conceptos fundamentales en la filosofía de Levinas es el de pasividad. Más allá de los antagónicos conceptos pasivo-activo, o mejor dicho más acá de ellos, ya que la significación de este concepto viene a expresar algo que es anterior, los precede. Pasividad originaria, radical, que no tiene origen ni comienzo, por ello mismo es pre-original, anárquica. Así, hace mover al sujeto en un plano diferente, ya no mantenerse en esa dicotomía que es asequible para el sujeto. La pasividad radical, es una pasividad anterior a toda libertad, voluntad, y resulta así, inaprensible. Se traduce en responsabilidad para con el otro, a la cual el sujeto no se puede sustraer, ya que se encuentra más allá de toda decisión propia. El Otro en la

\footnotetext{
${ }^{50}$ Cfr., LEVINAS, Emmanuel. Humanismo del otro hombre. Siglo veintiuno, Buenos Aires, 2003. p. 90.
} 
proximidad con el Mismo, evoca un llamado, frente al cual el mismo debe responder. El Otro se presenta como diferente, y la no indiferencia a aquello que es diferente se transcribe en responsabilidad. La aproximación a lo que es diferente, es una aproximación que me despoja de mis poderes sobre el otro, no hay intención de dominación, se está fuera del círculo de comodidad. De modo contrario, el movimiento propio de la libertad como lo caracteriza Levinas, solo hace posible la cercanía con aquello que se busca como igual, aquello que se anhela dominar, no es más que la supresión del Otro por la búsqueda de los propios intereses.

La proximidad al Otro, es una proximidad que se da, no en una relación de conocimiento. El conocimiento está bajo el poder de uno, en cambio, la proximidad al Otro excede mis poderes, y se da solamente en la responsabilidad para con él. El mismo no domina al otro, no logra emitir un saber sobre él, no lo tematiza, o como señala el autor, no lo hace Dicho. Moverse en lo Dicho, es querer adquirir un conocimiento sobre algo, volverlo tema, aprehenderlo, para con ello poder dominarlo. Pero, la subjetividad del Otro, excede la comprensión. Es invisibilidad, no se encuentra sometida al presente, a lo calculable, a lo manipulable, es diacronía, es decir, "desarreglo del tiempo rememorable" ${ }^{51}$. Fuera de todo cálculo, de toda representación, no admite que se lo encierre en unidad, que se lo totalice, y sigue manteniéndose como absolutamente Otro. Al no constituirse en fenómeno, se caracteriza por ser huella, ya que se encuentra al margen del presente $e^{52}$. Huella que posee de característico, el inquietar el estado presente sin ser aprehensible por la conciencia. Por ello mismo es, huella del exceso, debido a que supera la capacidad de la conciencia del sujeto para poder representarla. La huella, es propia de un ser que trasciende el mundo, es absoluto, y por eso puede dejar huella de su paso. Se encuentra más allá de la representación.

El Otro, se presenta al Mismo, como extranjero, sin pertenencia a un lugar, como indigente, necesitado de la respuesta al llamado que emite. El Mismo, que es un sujeto irremplazable, único, no puede desentenderse de ese llamado. Debe responder

\footnotetext{
${ }^{51}$ LEVINAS, Emmanuel. De otro modo que ser, o más allá de la esencia. Sígueme, Salamanca, 1995. p. 151.

${ }^{52}$ Cfr. LEVINAS, Emmanuel. De otro modo que ser, o más allá de la esencia. p. 56.
} 
por ese ser que se muestra con toda su carencia. Pero, no por ello, se encuentra en un estado superior, por el contrario, él se encuentra subordinado al Otro, es rehén de él. La responsabilidad es una responsabilidad irrecusable, y presenta al sujeto en una desnudez absoluta. El sujeto fuera de toda seguridad, se encuentra en su máxima vulnerabilidad. Expuesto a la violencia, se expone a la misma muerte. Sin embargo, el sujeto al encontrarse subordinado a una responsabilidad innegable, debe responder. $Y$ ello, no lo efectúa escuchando a un Yo, que le dice que actúe conforme a su libertad. La subjetividad del sujeto, en tanto pasividad, es anterior a toda decisión libre, previa al ejercicio de la voluntad.

Fuera de la persistencia en sí mismo, lo que individualiza a cada sujeto es el conjunto de acciones que ese sujeto hace y padece en su contexto mundano. Es así, como la identidad del sujeto hay que entenderla en términos de interacción, y éste, conforma su identidad a partir del Otro, a partir de la relación con el otro. Así se pasa a formular la identidad como, yo-soy-en-tanto-Otro. En la proximidad, el Otro se presenta ante uno formando así, un nosotros. Sin llegar a reducirlo a la mismidad, anulando su diferencia. Por ello también, esa dependencia que no se puede eludir con respecto al otro. El Otro configura la propia identidad. No depende solo de sí mismo lo que soy, sino en tanto soy en relación al Otro, en tanto responsabilidad para con el otro.

Pensar al Otro, en tanto sujeto diferente implica una salida de la órbita de seguridad del propio yo y con ello supone reconocer al Otro en su propia singularidad. El individuo no se caracteriza por pertenecer a un género, por reducir sus diferencias a un género, por el contrario, "su singularidad consiste en referirse cada una a sí misma"53. Pensamiento homogeneizante, que solo pretende reducir el Otro a lo Mismo ¿Acaso es temor a la imposibilidad de posesión del otro? La racionalidad calculadora solo tiene como efecto, un concepto del Otro como algo a la mano, como algo que puede volver tematizable, y a partir de ese conocimiento del otro, lo hace predecible y manipulable. Si el Otro, está fuera de los propios poderes, representa un riesgo. Constituye así un obstáculo con el cual se debe enfrentar. Conforme a ello, no

\footnotetext{
${ }^{53}$ LEVINAS, Emmanuel. Totalidad e infinito. Sígueme, Salamanca, 1999. p. 227.
} 
ve más que reducción, unidad. Lo Otro, como absolutamente otro, no resulta admisible.

Por el contrario, la subjetividad en tanto pasividad, es irreductible. No se deja abolir por la razón totalizadora. Y ello, debido a que escapa a todo contenido de la conciencia, no es dato. Fuera de toda estrategia de apropiación y dominación, la subjetividad persiste en su diferencia, sin mantener un desinterés hacia el Otro. El desinterés es siempre interés. Es la forma perenne del Otro, que siempre va a estar fuera del propio poder. Incapacidad del sujeto a no poder no responder por él, ya que la negación es así mismo una respuesta.

La ruptura con un pensamiento habituado a reducir todo a fenómeno, aprehensión, la plantea Levinas, cuando señala que la subjetividad del sujeto es exceso, exceso con respecto a la conciencia ${ }^{54}$. No fenómeno, que pasa por encima del presente, desborda la ubicación en un tiempo rememorable. Por ello, es diacronía, tiempo trastocado, alterado con respecto a un tiempo, que es más bien, sincrónico.

La relación asimétrica con el Otro, hace ver como el Mismo y el Otro son seres únicos e irremplazables que no pueden ser reunidos en ningún género. Levinas refiere a ello, como la relación metafísica, en la que el Otro no puede ser reducido al Mismo. Así, el otro es trascendencia, una realidad infinitamente distinta. "Es huella del infinito" ${ }^{\prime 55}$. Esto supone una nueva comprensión del sujeto, definido no en términos de poder, sino de pasividad, de transcendencia o apertura a la exterioridad.

Por consiguiente, la relación con el otro, en tanto responsabilidad para con el otro, no ha de pensarse como el Otro absorbiéndose en el Mismo, reduciéndose a la mismidad del sujeto, sino en tanto acogimiento del uno al Otro, y en tanto dar.

Como se mencionó al comienzo Levinas denuncia una reducción identitaria del Mismo hacia el Otro, a partir de la relación saber-ser. Cabe preguntar, ¿es posible acceder al Otro más allá de un pensamiento que lo piensa, y aun así, no perder la relación? Dicho interrogante puede ser resuelto siguiendo al autor a partir de la consideración de la preeminencia de la relación ética frente a la ontología.

\footnotetext{
${ }^{54}$ Cfr., LEVINAS, Emmanuel. Humanismo del otro hombre. p. 98.

${ }^{55}$ Op. Cit. LEVINAS, Emmanuel. De otro modo que ser, o más allá de la esencia. p. 57.
} 


\section{Preeminencia de la relación ética.}

Levinas presenta en Totalidad e infinito la primacía de la metafísica por sobre la ontología. La metafísica representaría la forma de la relación ética, la cual permite no meramente una relación cognoscitiva sino de reconocimiento del Otro. El filósofo lituano, describe dicha relación de la siguiente manera:

\footnotetext{
El saber o la teoría significa primeramente una relación tal con el ser, que el ser cognoscente deja manifestarse al ser conocido respetando su alteridad y sin marcarlo en modo alguno con esta relación de conocimiento. En este sentido, el deseo metafísico seria la esencia de la teoría ${ }^{56}$.
}

La propuesta ética de Levinas concibe la ética como filosofía primera, donde el énfasis no se encuentra puesto en la verdad si no en el otro, representa así un nuevo pensamiento donde se considera la responsabilidad por el otro y su acogida en la apertura. Conforme a esto, cuestiona los fundamentos de la racionalidad occidental.

Este tipo de racionalidad procede en términos no relacionales, ya que el conocimiento se resumiría en un conocimiento egológico. El movimiento libre del Mismo, procede mediante la neutralización del Otro en esa búsqueda de la unidad o identidad. Es así como, "conocer viene a ser aprehender el ser a partir de nada o llevarlo a la nada, quitarle su alteridad" ${ }^{27}$. En este sentido, conocer, significa adecuar al Otro al Mismo, adaptación del Otro a la verdad de mi ser.

Contrariamente, en Levinas se puede entrever que toda la realidad es ontológicamente relacional. Es por ello, que el conocimiento de lo humano no surge de sí mismo, la verdad no se encuentra en la mismidad, a la manera socrática, sino en la pluralidad. La relación ética, ya no se basa en la idea del sujeto que reduce al Otro a sus categorías cognitivas, sino que lo preponderante es que la relación con el Otro domina la comprehensión del $\operatorname{ser}^{58}$. El Otro no es reductible a categorías mentales del sujeto porque precede y excede al Mismo, conmoviéndolo antes de la intervención de

\footnotetext{
${ }^{56}$ Op. Cit. LEVINAS, Emmanuel. Totalidad e infinito. p.66.

${ }^{57}$ Cfr., LEVINAS, Emmanuel. Totalidad e infinito. p. 68.

${ }^{58}$ Ibíd., p. 71.
} 
la voluntad. Esa conmoción es la que se traduce por sensibilidad. Lo que significa no poder dejar de responder frente al Otro, frente a su sufrimiento, frente a su súplica.

\section{Consideraciones conclusivas}

Lo que caracteriza según Foucault al régimen de disciplina es la manera en que la coacción mediante la violencia ha sido sustituida, por un cuerpo médico administrativo, compuesto por expertos científicamente preparados $y$, en definitiva, por la exhibición de poder mediante el despliegue de una tecnología y técnicas basadas en un conocimiento detallado de sus objetivos. La relación saber-poder ejercería un papel fundamental en este proceso. Ello resultaría así, ya que, según este autor, el poder disciplinario es anomizante y normalizador ${ }^{59}$, es decir, establece lo diferente, aquello inclasificable, lo que aparece como lo anómico, lo anormal, y a la vez establece acorde a ellos los sistemas disciplinarios para normalizarlos. Es así, como el saber disciplinario hace aparecer los "residuos", aquello inasimilable, inadecuado a la regla. Consecuentemente se producen cuerpos sujetos, los cuales se ven sometidos constantemente por ese saber-poder mediante la utilización de diversas técnicas sobre los cuerpos, que tienen como fin neutralizar al enfermo, volverlo dócil y por consiguiente se enaltece la figura del médico y su autoridad se vuelve absoluta.

Respecto a esa neutralización del Otro, Levinas denuncia su reducción a las categorías cognitivas del Mismo, lo que se efectúa es un ajuste a las categorías mentales del sujeto producto de un abordaje meramente racional promovido por la ontología.

La ontología como conocimiento, suscita esa libertad del yo, que tiene como objeto la identidad y la homogeneidad. Tal como lo presenta Foucault, la construcción de cuerpos sujetos a partir de un saber-poder que se entroniza a partir de la docilidad del enfermo, retorna a esa racionalidad cuyo fin es la reducción del Otro a conceptos, reducción del Otro al Mismo.

${ }^{59}$ Cfr., Foucault, Michel. El poder psiquiátrico. Fondo de Cultura Económica, Bs. As, 2014. p. 76. 